\title{
NOVAS OCORRÊNCIAS DE HETERODERMIA (LÍQUENS- PYXINACEAE) NO RIO GRANDE DO SUL, BRASIL ${ }^{1}$
}

\author{
Sionara Eliasaro ${ }^{2}$ \\ Mariana Fleig ${ }^{3}$
}

Recebido em 29.10.92. Aceito em 13.01.94.

RESUMO - Novas ocorrências de Heterodermia (Líquens - Pyxinaceae) no Rio Grande do Sul, Brasil. Heterodermia hypoleuca (Muhl.) Trev. e H. squamulosa (Degel.) W. Culb. são citadas como novas para o Brasil, e H. corallophora (Taylor) Skorepa, H. microphylla (Kuork.) Skorepa, H. trichophora (Kurok.) S. Eliasaro (nova combinação) são anexadas à flora conhecida do Estado do Rio Grande do Sul.

Palavras-chave: Heterodermia, líquens, florística.

\begin{abstract}
New occurrence of Heterodermia (Lichens - Pyxinaceae) in Rio Grande do Sul, Brasil). Heterodermia hypoleuca (Muhl.) Trev. and H. squamulosa (Degel.) W. Culb. are reported as new to Brasil, and $H$. corallophora (Taylor) Skorepa, $H$. microphylla (Kuork.) Skorepa, $H$ : trichophora (Kurok.) S. Eliasaro (new combination) are added to the known flora of Rio Grande do Sul state.
\end{abstract}

Key-words: Heterodermia, lichens, floristics.

\section{Introdução}

O gênero Heterodermia foi objeto deste estudo, visando o conhecimento e distribuição das espécies no Estado do Rio Grande do Sul (Eliasaro 1992).

Existem poucos dados sobre o gênero para o Brasil. Para o Rio Grande do Sul, Lynge (1924) relata a ocorrência de 4 espécies, baseado em coletas de Malme no sul do Brasil. Osório \& Homrich (1978) e Osório et al. (1980, 1982, 1983) citam 12

\footnotetext{
'Parte de dissertação de Mestrado desenvolvida no Depto. Botânica, Universidade Federal do Rio Grande do Sul - bolsa CAPES.

²Departamento de Botânica, S. Biológicas, Universidade Federal do Paraná, Caixa Postal 19031, 81531970, Curitiba-PR, Brasil.

${ }^{3}$ Departamento de Botânica, Universidade Federal do Rio Grande do Sul, Av. Paulo Gama, 110, 90049, Porto Alegre-RS, Brasil.
} 
espécies para o Rio Grande do Sul. Fleig (1990), acresce duas novas ocorrências para o Estado.

O objetivo deste trabalho é relatar as novas ocorrências do gênero Heterodermia no Rio Grande do Sul.

\section{Material e métodos}

Foram analisadas exsicatas do gênero Heterodermia coletadas em diversas regiões fisiográficas do Rio Grande do Sul, depositadas no herbário do Departamento de Botânica da Universidade Federal do Rio Grande do Sul (ICN). Também analisaram-se exemplares-tipo dos herbários Uppsala University, Suécia (UPS) e Farlow Reference Library and Herbarium of Criptogamic Botany, Harvard University, USA (FH). Além do material de herbário foram feitas observações em campo e novas coletas incorporadas ao herbário ICN.

Realizou-se análise morfológica externa e anatômica do talo e apotécio sob estereoscópio e microscópio óptico. A análise química constou de teste de coloração (KOH 10\%), usual em liquenologia, de microcristalografia segundo Hale (1974) e submeteu-se à cromotografia de camada delgada parte do material examinado. $\mathrm{O}$ pròcedimento básico seguiu White e James (1985) sendo o extrato acetônico de cada exemplar aplicado, juntamente com extrato padrão de atranorina, ácido norstíctico e ácido salazínico, em placa de vidro recoberta por camada fina de sílica gel. O sistema de solventes utilizado foi benzeno-dioxano-ácido acético (180:45:5). As duas útimas técnicas foram desenvolvidas sob orientação da Prof. ${ }^{a}$ Neli K. Honda, no laboratório de Química da Universidade Federal do Mato Grosso do Sul.

\section{Resultados e comentários}

Na análise das exsicatas do gênero Heterodermia procedentes do Rio Grande do Sul, foi possível constatar a ocorrência de 19 espécies e uma subespécie (Eliasaro, 1992). Cinco táxons constituem novos registros para o Estado, sendo dois destes novidades também para o Brasil.

As cinco espécies do gênero Heterodermia, novas para o Rio Grande do Sul, objeto deste trabalho, foram coletadas na região da Serra Geral. $H$. corallophora e $H$. trichophora foram encontradas em áreas de maior altitude, enquanto as demais, na encosta da Serra, ocorrendo $H$. squamulosa e $H$. microphylla também em áreas rochosas de menor altitude na metade sul do Estado.

Heterodermia corallophora (Taylor) Skorepa, Bryologist 75:49. 1972. Basinômio: Parmelia corallophora Taylor, Lond. J. Bot. 6:167. 1847. Tipo: Peru, Casapi, Mathews (FH - Holótipo visto).

Talo rosetado, adpresso ao substrato em toda sua extensão. Numerosos isídios, simples a bifurcados ou aglomerados, principalmente laminais. Superfície inferior 
não corticada. Córtex superior irregular, camada de algas subcontínua. Não observada formação de apotécios. Córtex superior, medula e superfície inferior $\mathrm{K}+$ amarelo; atranorina e zeorina.

Material examinado - Rio Grande do Sul: Cambará do Sul, Parque Nacional dos Aparados da Serra, Itaimbezinho, 21-IX-1980, Gruninger \& M. Fleig (ICN 54132); Esmeralda, Estação Ecológica de Aracurí, 29-III-1982, M. Fleig 1552 (ICN 54745); 22-I-1984, M. Fleig 2295a (ICN 55967).

Comentários - Heterodermia corallophora desenvolve-se sobre córtex de árvore em locais abertos, em altitudes superiores a $900 \mathrm{~m}$.

Espécie facilmente distinguível por ser a única no Estado a desenvolver isídios.

Kurokawa (1962) relata a presença de um pigmento amarelo-limão próximo ao ápice. Esta pigmentação não foi observada nos exemplares ocorrentes no Estado, como também não o foi no exemplar-tipo.

Heterodermia hypoleuca (Muhl.) Trev., Atti Soc. Ital. Sci. Nat. 11:615. 1868.

Basinômio: Parmelia hypoleuca Muhl., Cat. Pl. Amer. Sept. 1813.

Tipo: América do Norte, Muhlberg 33-2 (H - não visto).

Talo rosetado, adpresso ao substrato em toda sua extensão. Superfície inferior não corticada. Sem propágulos vegetativos. Córtex superior irregular, camada de algas subcontínua. Apotécio laminal. Esporos pouco a não constritos no septo, 24-33 x 11-13 $\mu \mathrm{m}$, com esporoblatídeos. Córtex e medula $\mathrm{K}+$ amarelo: atranorina e zeorina. Material examinado - Rio Grande do Sul: Salvador do Sul, Colégio Santo Inácio, $27-$ IV-1990, S. Eliasaro 874 (ICN 72986); S. Eliasaro 876 (ICN) 72988); 26-IV-1991, S. Eliasaro 911 (ICN 73121).

Comentários - Heterodermia hypoleuca desenvolve-se sobre córtex de árvores em locais úmidos.

Kurokawa (1962) inclui esta espécie na série Speciosae, caracterizada por produzir esporos sem esporoblastídeos. Swinscow \& Krog (9176), na análise dos esporos do holótipo, encontraram esporoblastídeos em um dos esporos, e verificaram que alguns exemplares do Este Africano formam, ocasionalmente, esporos com esporoblastídeos. Citam a ocorrência de exemplares onde estes são numerosos, o que está em pleno acordo com nosso material.

Vainio (1890) e Lynge (1924) fazem a citação desta espécie para o Brasil; Kurokawa (1962) porém, notifica ter sido esta espécie mal interpretada, tratando-se na realidade de exemplares de $H$. flabellata, que se distingue por possuir rizinas pretas esquarrosas, esporos maiores e apresentar pigmento amarelo ou ocráceo na superfície inferior. Logo, esta é a primeira citação de $H$. hypoleuca para o Brasil.

Heterodermia microphylla (Kurok.) Skorepa, Bryologist 75(4):490. 1972.

Basiônimo: Anaptychia hypoleuca (Ach) Mass. var. microphylla Kurokawa, J. Jap. Bot. 34:123.1959.

Tipo: Japão, Honshu, prov. Shinamo, Sakakita-mura, Higashi-Chikumagum, em rochas, Yamazaki, 28-VII-1953 (TNS- não visto). 
Talo rosetado, adpresso ao substrato em toda sua extensão.

Escamas marginais numerosas, sorediadas, revolutas, ramificadas, filiformes. Superfície inferior não cortificada. Córtex superior regular, camada de algas contínua. Não observada formação de apotécios. Córtex e medula K+ amarelo; atranorina e zeorina.

Material examinado - Rio Grande do Sul: Bagé, Casa de Pedra, BR 153 Km 193, 27 XII-1990, M. Fleig 4171 (ICN 72835); Cambará do Sul, Parque Nacional dos Aparados da Serra, Itaimbezinho, 29-X-1988, M. Fleig 3665 (ICN 73356); Mariana Pimentel, Reserva da ASPRAM, 17-IV-1982, M. Fleig 1561 (ICN 54753)

Comentários - Esta espécie desenvolve-se sobre córtex de arbustos e árvores, em locais úmidos, mata aberta.

Kurokawa (1962) cita a produção ocasional de ácido norstíctico e de ácido salazínico para esta espécie; no entanto, em nenhum dos exemplares analizados observou-se a formação de depsidonas.

Espécie geralmente citada para outros continentes; Kalb (1984) cita-a para Mato Grosso do Sul. Não analisamos o exemplar-tipo e também não consultamos material de outros herbários; a identificação de nosso material foi baseada nas descrições de Kurokawa (1962) e Swinscow \& Krog (1976).

Heterodermia squamulosa (Degel.) W. Culb., Bryologist 69:484. 1966. Basiônimo: Anaptychia squamulosa Degel., Ark. Bot. 30a (3):76. 1941. Tipo: USA, Carolina do Norte, Great Smokey, Degelius (US - não visto).

Talo rosetado, adpresso ao substrato em toda sua extensão.Escamas numerosas, principalmente marginais, eretas, digitiformes, de cilíndricas a levemente achatadas, dorsiventrais. Superfície inferior não corticada. Córtex superior irregular, camada de algas subcontínua. Apotécio laminal. Esporos 39-49 X 17-21 $\mu \mathrm{m}$, com esporoblastídeos. Córtex e medula $\mathrm{K}+$ amarelo; atranorina e zeorina.

Material examinado - Rio Grande do Sul: Bagé, Br 153 Km 84, 29-V-1985, C. Grabauska 81 (ICN 73355); Casa de Pedra, M. Fleig 4122 (ICN 72398); Barracão, Espigão, 30-X-1988, M. Fleig 3676 (ICN 73357); Canela, leste da cidade, 17-IV1988, M. Fleig 3190 (ICN 70942); Guaíba, Chácara Matzenbacher, 22-IV-1985, M. Fleig 2625 (ICN 70125); São Francisco de Paula, Floresta Nacional do IBDF, 12-IX1980, M. Fleig 1154 (ICN 54345);Taquarí, Morro da Carapuça, 06-IV-1985, C. Grabauska \& P. Balbueno 24 (ICN 55955); Viamão, Parqu Saint Hilaire, 24-IV-1982, M. Fleig 1588 (ICN 54883).

Comentários - Heterodermia squamulosa desenvolve-se sobre córtex de árvores e sobre rochas, em mata aberta ou beira mata.

Espécie distinta das demais ocorrentes no Estado por possuir escamas tanto marginais quanto laminais. Muitas delas, á primeira vista dão a impressão de isídios, por serem parcialmente cilíndricas.

Esta espécie não foi anteriormente citada para o Brasil. Não fizemos análise do exemplar-tipo e também não analisamos exemplares de outros herbários. A identificação desta espécie foi feita tendo como base a descrição de Kurokawa (1962). 
Heterodermia trichophora (Kurokawa) S. Eliasaro, nova combinação.

Basiônimo: Anaptychia trichophora Kurokawa, Beih. Nova Hedwgia 6:44. 1962.

Tipo: Brasil, Rio de Janeiro, Serra do Picu, 11-XII-1886, Schenk 4436 (UPS Holótipo visto).

Talo rosetado, aderido ao substrato pela região basal. Superfície inferior não corticada. Sorais apicais na superfície inferior dos lobos, sorédios subgranulosos. Córtex superior irregular, camada de algas subcontínua, medula fina a inexistente. Apotécio de apical a subapical, margem ciliada, receptáculo também ciliado, cílios curtos, menores que 1,0mm de comprimento. Esporos 35-40 X 15-18 $\mu \mathrm{m}$, com esporoblastídeos. Córtex e medula $\mathrm{K}+$ amarelo; atranorina e zeorina.

Material examinado - Rio Grande do Sul: Cambará do Sul. Parque Nacional dos Aparados da Serra, Itaimbezinho, 29-X-1980, M. Fleig 1220 (ICN 54411); Fortaleza dos Aparados, 29-X-1983, M. Fleig 2200 (ICN 55553).

Comentários - Heterodermia trichophora desenvolve-se em córtex de ramos fínos de arbustos e árvores.

Espécie assemelhada a $H$. comosa pela presença de cílios no apotécio e aspecto morfológico do talo, desta diferindo pela ausência de cílios na superfície superior do talo.

Citada unicamente para o Brasil (Minas Gerais) e Bolívia (Kurokawa 1962).

\section{Agradecimentos}

Expressamos nossos agradecimentos aos curadores dos herbários UPS e FH pelo empréstimo dos exemplares-tipo; à Prof ${ }^{a}$ Dr $^{a}$ Rosa T. Guerrero pela orientação e à Prof ${ }^{\wedge}$ Neli k. Honda pela orientação na análise química.

\section{Referências bibliográficas}

Eliasaro, S. 1992. Líquens do gênero Heterodermia (Pyxinaceae-Ascomycotina) no Rio Grande do Sul. Porto Alegre: Universidade Federal do Rio Grande do Sul. Dissertação de Mestrado.

Fleig, M. 1990. Líquens da Estação Ecológica de Aracurí. Novas ocorrências no Rio Grande do Sul. Iheringia (Bot.) 40: 121-125.

Hale, M. E. 1974. The Biologh of Lichens. Edward Arnold.

Kalb, K. 1984. Lichens Neotropici. 2-8 (41-350).

Kurokawa, S. 1962. A monografh of the genus Anaptychia. Beih. Nova Hedwigia, 6:1-115.

Lynge, B. 1924. On South American Anaptychiae and Physciae. Skr. vid. Math-Naturvidensk. Kl., 2:1-49.

Osório, H. S. \& Homrich, M. H. 1978. Contribution to lichen flora of Brazil IV: Lichens from Sourthern Rio Grande do Sul. Bryologist, 81 (3): 452-454.

Osório, H. S. et al.1980. Contribution to the lichen flora of Brazil VII. Lichens from Montenegro and Triunfo, Rio Grande do Sul State. Com. Bot. Mus. Hist. Nat. Montevideo, 4(62) :1-8.

Osório, H. S. et. al. 1982. Contribution to the lichen flora of Brazil X: Lichens from Guaíba, Rio Grande do Sul State. Phytologia, 51(7): 479-483.

Osório, H. S. et al. 1983. Contribution to the lichen flora of Brazil XII: Lichens from São Jerônimo, Rio Grande do Sul State. Phytologia, 53 (3):138-140. 
Swinscow, T. D. V. \& Krog, H. 1976. The fenus Anaptychia and Heterodermia in East Africa. Lichenologist, 8:103-138.

Vainio, E. A. 1890. Étude sur la classification naturelle et la morphologie des lichens du Brésil. Acta Soc. Fauna Fl. Fenn., 7(1):1-247,

White, J. \& James, P. W. 1985. A new guide to microchemical techniques for the identification of the lichen substances. British Lichen Society; Bulletin 57 (Suppl.). 